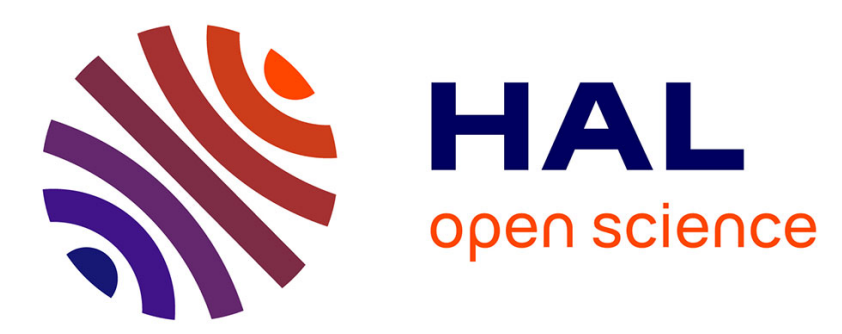

\title{
Étude en microscopie électronique des transitions de phase dans les oxydes de vanadium de composition V2O3 et (V1-xCrx)2O3
}

R. Ayroles, Christian Roucau, Jean-Claude Launay, Michel Pouchard

\section{To cite this version:}

R. Ayroles, Christian Roucau, Jean-Claude Launay, Michel Pouchard. Étude en microscopie électronique des transitions de phase dans les oxydes de vanadium de composition V2O3 et (V1-xCrx)2O3. Journal de Physique Colloques, 1976, 37 (C4), pp.101-104. 10.1051/jphyscol:1976415 . jpa-00216529

HAL Id: jpa-00216529

https://hal.science/jpa-00216529

Submitted on 1 Jan 1976

HAL is a multi-disciplinary open access archive for the deposit and dissemination of scientific research documents, whether they are published or not. The documents may come from teaching and research institutions in France or abroad, or from public or private research centers.
L'archive ouverte pluridisciplinaire HAL, est destinée au dépôt et à la diffusion de documents scientifiques de niveau recherche, publiés ou non, émanant des établissements d'enseignement et de recherche français ou étrangers, des laboratoires publics ou privés. 


\title{
ÉTUDE EN MICROSCOPIE ÉLECTRONIQUE DES TRANSITIONS DE PHASE DANS LES OXYDES DE VANADIUM DE COMPOSITION $\mathrm{V}_{2} \mathrm{O}_{3}$ et $\left(\mathrm{V}_{1-x} \mathrm{Cr}_{x}\right)_{2} \mathrm{O}_{3}$
}

\author{
R. AYROLES et C. ROUCAU \\ Laboratoire d'Optique Electronique du C. N. R. S. \\ 29, rue Jeanne Marvig, 31055 Toulouse Cedex, France \\ J. C. LAUNAY et M. POUCHARD \\ Laboratoire de Chimie du Solide du C. N. R. S. \\ Université de Bordeaux I, 351, cours de la Libération, 33405 Talence, France
}

\begin{abstract}
Résumé. - Les transitions de phase vers les basses températures dans le sesquioxyde de vanadium pur ou dopé donnent une phase antiferromagnétique isolante composée de domaines cristallographiques. La configuration de ces domaines a pu être déterminée au microscope électronique en comparant leurs images et les diagrammes de diffraction correspondants. Par ailleurs, des expériences effectuées à tension accélératrice élevée $(1500 \mathrm{kV})$, afin de bénéficier d'une meilleure pénétration des électrons, ont permis d'enregistrer des phénomènes évolutifs au voisinage de la transition. On a observé en particulier que ces domaines caractérisant la phase antiferromagnétique isolante se subdivisent avant de đisparaître à la transition. Ces phénomènes évoluent réversiblement lorsqu'on abaisse la température du cristal.
\end{abstract}

\begin{abstract}
Electron microscope study of phase transition in vanadium oxides with composition $\mathrm{V}_{2} \mathrm{O}_{3}$ and $\left(\mathrm{V}_{1-x} \mathrm{Cr} x\right)_{2} \mathrm{O}_{3}$. The phase transitions at low temperatures in the vanadium sesquioxide, pure or doped, take on antiferromagnetic insulator phase with crystallographic domains. The configuration of these domains was investigated with the electron microscope by comparing their images and corresponding diffraction patterns. The better penetration of high voltage $(1500 \mathrm{kV})$ electrons improved observation of the evolution of phenomena near the phase transition. In particular, the domains characterizing the antiferromagnetic phase were seen to subdivise before and disappear at the transition. The phenomena develop reversibly when the crystal temperature is reduced.
\end{abstract}

1. Introduction. - La mise au point d'une technique de préparation de monocristaux de sesquioxyde de vanadium $\mathrm{V}_{2} \mathrm{O}_{3}$ purs ou dopés [1] destinés à l'observation au microscope électronique, permet maintenant d'enregistrer les effets des transitions de phase obtenues en faisant varier la température des échantillons. En utilisant des cristaux de très bonne qualité [2], nous avons pu suivre l'évólution des phénomènes au voisinage des températures de transition.

D'une manière générale, la transition vers les basses températures est caractérisée par la formation de domaines cristallographiques plus ou poins étendus dans les cristaux, et nous allons d'abord indiquer quelle peut être leur configuration. Nous verrons ensuite comment s'opère la formation de ces domaines en effectuant des observations in situ sous tension accélératrice élevée où le gain en pénétration des électrons est avantageux.

2. Configuration des domaines apparus au cours des transitions à basse température. - Divers auteurs ont déjà montré que la transition de phase du sesquioxyde de vanadium pur ou dopé au chrome entraîne la formation de domaines cristallographiques [1] [3]. Ceux-ci peuvent être de plusieurs types comme l'ont par ailleurs observé Hayashi, Van Landuyt et Amelinckx [4] sur des cristaux de $\mathrm{VO}_{2}$.

Le cas le plus simple que nous ayons rencontré est illustré par la figure 1. Cette figure représente les clichés en champ sombre d'un cristal de $\left(\mathrm{V}_{1-x} \mathrm{Cr}_{x}\right)_{2} \mathrm{O}_{3}$. $(x=0,024)$, pris avant et après la transition I-AFI (isolant-antiferromagnétique isolant) obtenue pour $T \sim 180 \mathrm{~K}$ [2]. A $110 \mathrm{~K}$ les domaines sont stabilisés et leurs interfaces s'identifient aux plans cristallographiques $(2 \overline{1} 1)_{\mathrm{M}}$ dans la maille monoclinique ou $(11 \overline{2} 0)_{\mathrm{H}}$ dans la maille hexagonale dont elle dérive. Comme ces domaines présentent des contrastes identiques de part et d'autre des interfaces, cela quelle que soit la direction du faisceau incident par rapport au réseau cristallin, on peut en conclure que leur structure et leur orientation les uns par rapport aux autres sont partout les mêmes. Un modèle cristallographique a été proposé pour interpréter ce résultat dû au fait que les atomes de vanadium peuvent se déplacer, au cours de la transition, dans différents plans du type $(11 \overline{2} 0)_{\mathrm{H}}[1]$. Les plans des interfaces peuvent également s'identifier 

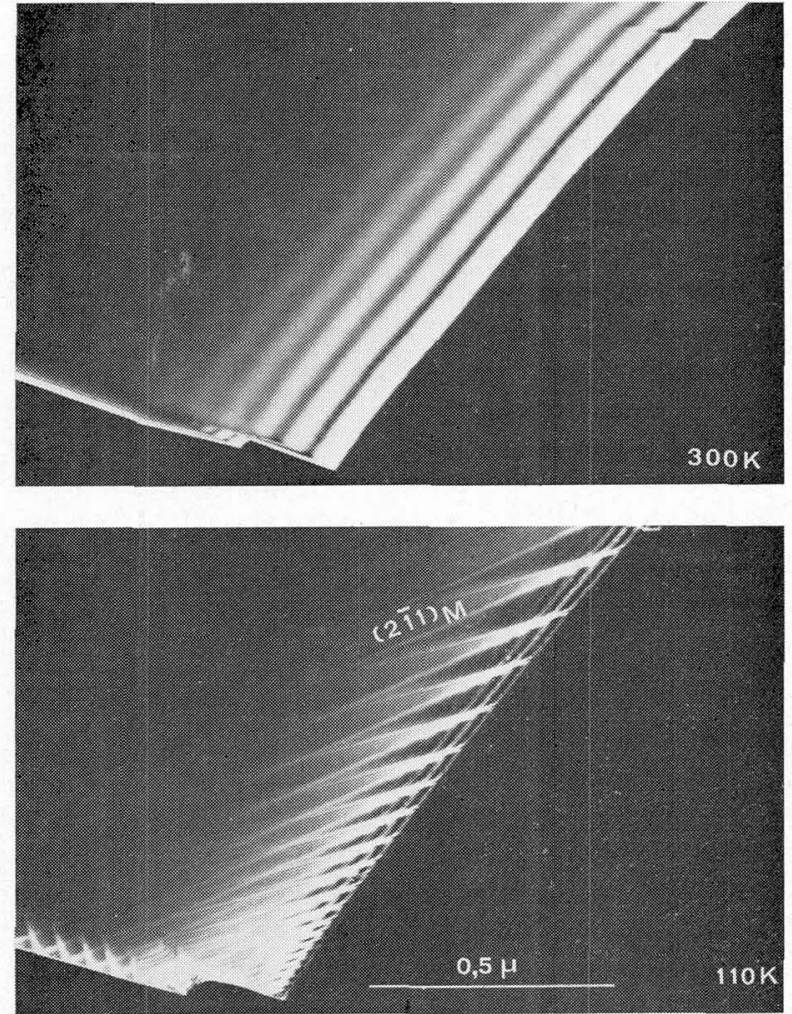

FIG. 1. - Images en champ sombre d'un cristal $\left(\mathrm{V}_{1-x} \mathrm{Cr}_{x}\right)_{2} \mathrm{O}_{3}$ avec $x=0,024$, obtenues sous une tension de $100 \mathrm{kV}$ avant et après la transition I-AFI. On note les interfaces situées dans les plans $(\overline{2} \overline{1})_{\mathrm{M}}$.

à une famille $(10 \overline{10})_{\mathrm{H}}$ [3]. Par ailleurs, nous avons mis en évidence dans un cristal de $\mathrm{V}_{2} \mathrm{O}_{3}$, un troisième type d'interface qui se situe dans des plans $(\overline{101})_{\mathrm{M}}$ ou $(0001)_{\mathrm{H}}$. Nous proposons un modèle montrant en définitive que la maille élémentaire monoclinique se déduit, d'un domaine à l'autre, par une rotation de $\frac{2 \pi}{3}$ autour de l'axe $\mathrm{C}_{\mathrm{H}}$ de la maille hexagonale primitive.

Les images en champ sombre reproduites sur la figure 2 correspondent au cas le plus fréquemment observé. C'est ici un cristal de $\mathrm{V}_{2} \mathrm{O}_{3}$ stoechiométrique dont la transition M-AFI (métal-antiferromagnétique isolant) a lieu vers $150 \mathrm{~K}$. Dans la phase AFI, on remarque la présence, dans le cristal, de domaines bien distincts qui présentent alternativement le long de l'arête, des contrastes très différents. Les diagrammes de diffraction ne permettent pas de faire apparaître des différences de structure. Par contre, ils montrent que ces domaines sont légèrement désorientés les uns par rapport aux autres. Dans le cas de la figure 2, des diagrammes de diffraction ont été obtenus respectivement sur la région claire de l'image (cliché b) où le domaine est pratiquement en position de Bragg sur le spot du second ordre $(2 \overline{2} 0)_{\mathrm{M}}$, et sur la région sombre (cliché c) où les lignes de Kikuchi ont subi un déplacement d'une quantité voisine de $\frac{1}{2} g_{1} \overline{1} 0$ par rapport au spot $2 \overline{2} 0$ dans la direction [1 $\overline{1} 0]$. La comparaison de
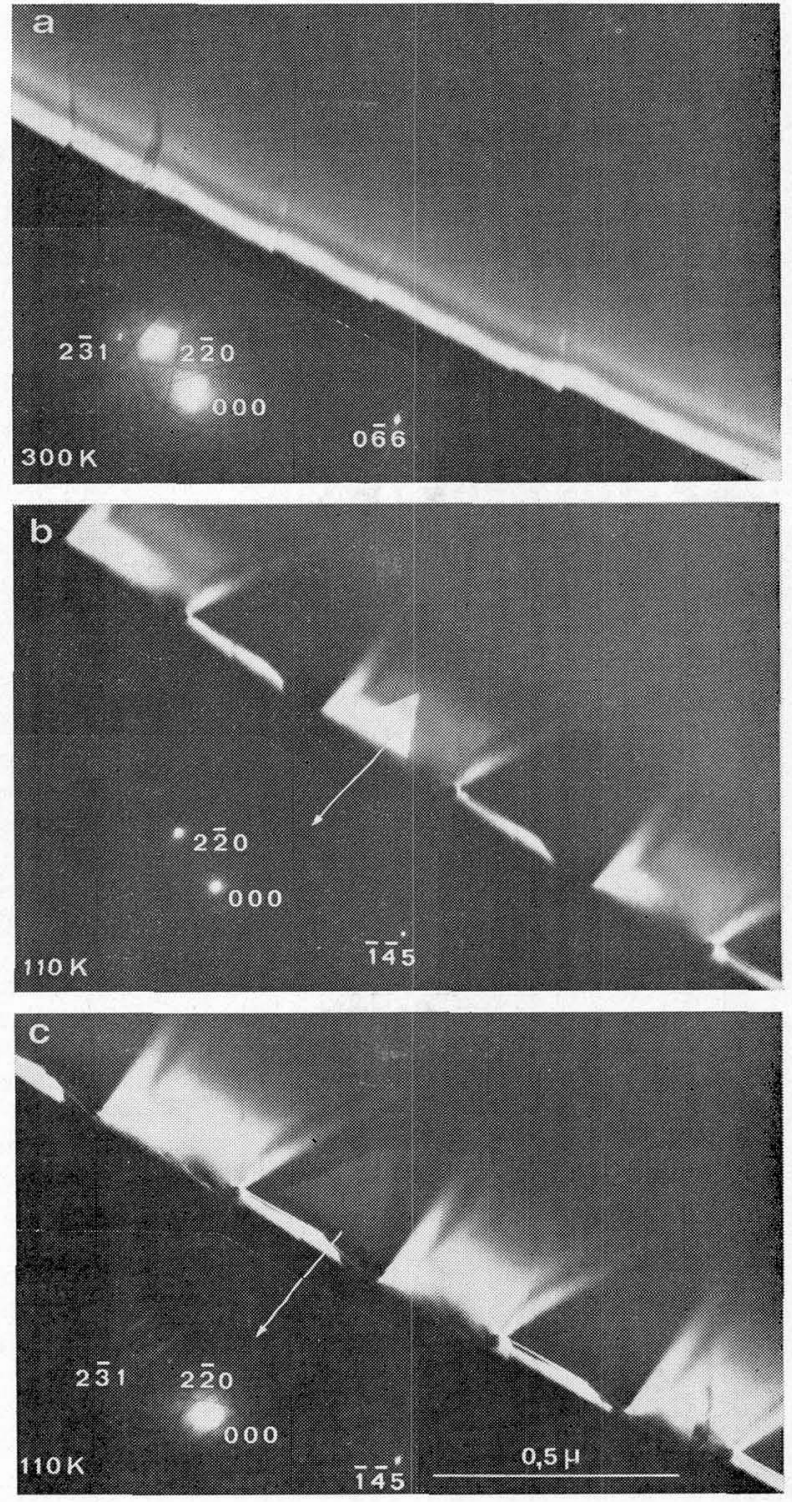

FIG. 2. - Effet de la transition M-AFI dans un cristal de $\mathrm{V}_{2} \mathrm{O}_{3}$ entraînant la formation de domaines légèrement désorientés.

ces diagrammes montre que lâ rotation entre les domaines consécutifs est de l'ordre de l'angle de Bragg $\theta_{1 \overline{1} 0}=4,4 \times 10^{-3} \mathrm{rad}$.

3. Evolution des phénomènes pendant le passage à la transition observé à haute tension accélératrice. Des expériences effectuées sous tension accélératrice élevée $(1500 \mathrm{kV})$ pour bénéficier d'une meilleure pénétration des électrons, nous ont permis d'enregistrer les effets produits par le changement de phase. La figure 3 représente un montage photographique qui montre, sur une région déterminée de l'échantillon, quelques étapes au cours desquelles s'opère la transition $\mathrm{M}$-AFI, vers $150 \mathrm{~K}$, dans un cristal de $\mathrm{V}_{2} \mathrm{O}_{3}$ stoechiométrique. Ces images, ainsi que celles reproduites sur la prochaine figure, ne sont pas de bonne 

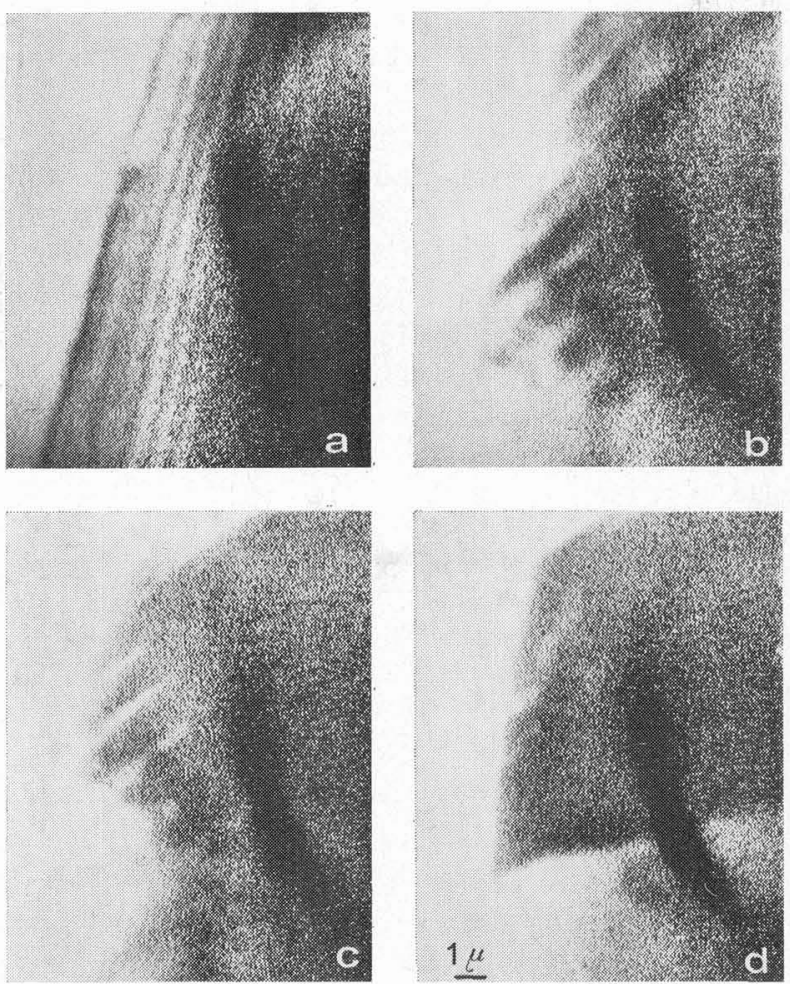

FIG. 3. - Transition M-AFI dans un cristal de $\mathrm{V}_{2} \mathrm{O}_{3}$, obtenue par abaissement de la température sous une tension de $1500 \mathrm{kV}$.

qualité, d'une part parce qu'elles ont été obtenues à partir d'un enregistrement effectué sur magnétoscope, d'autre part parce que la variation de température entraîne une dérive importante de l'objet, ce qui interdit la prise directe de photographies au microscope électronique. Néanmoins, les cristaux utilisés sont de très bonne qualité ; nous avons montré par ailleurs que leur résistivité décroît d'un facteur $10^{10}$ à la transition [2]. Malgré ce caractère abrupt de la transition, on observe une évolution des images en abaissant lentement la température du cristal. L'image $a$ correspond à l'état métallique du cristal. Lorsque la température diminue, il apparaît soudainement des microdomaines (cliché b) qui tendent à se regrouper (cliché c) pour former finalement un domaine relativement étendu et stable (cliché d) à une température nettement inférieure à la température de transition (quelques dizaines de $\mathrm{K}$ ).

Les images reproduites sur la figure 4 montrent l'évolution inverse du phénomène lorsque la température croît au voisinage du point de transition AFI-M. Le domaine relativement important sur le cliché a laissé la place à des micromacles dans la partie mince du cristal (cliché b). Ces micromacles s'ordonnent tandis que leur nombre augmente (cliché c). Enfin, elles disparaissent brutalement et l'on obtient l'image classique d'un cristal en forme de coin avec des franges encore perturbées (cliché d) qui deviennent pratiquement parfaites au fur et à mesure que la température continue à augmenter.
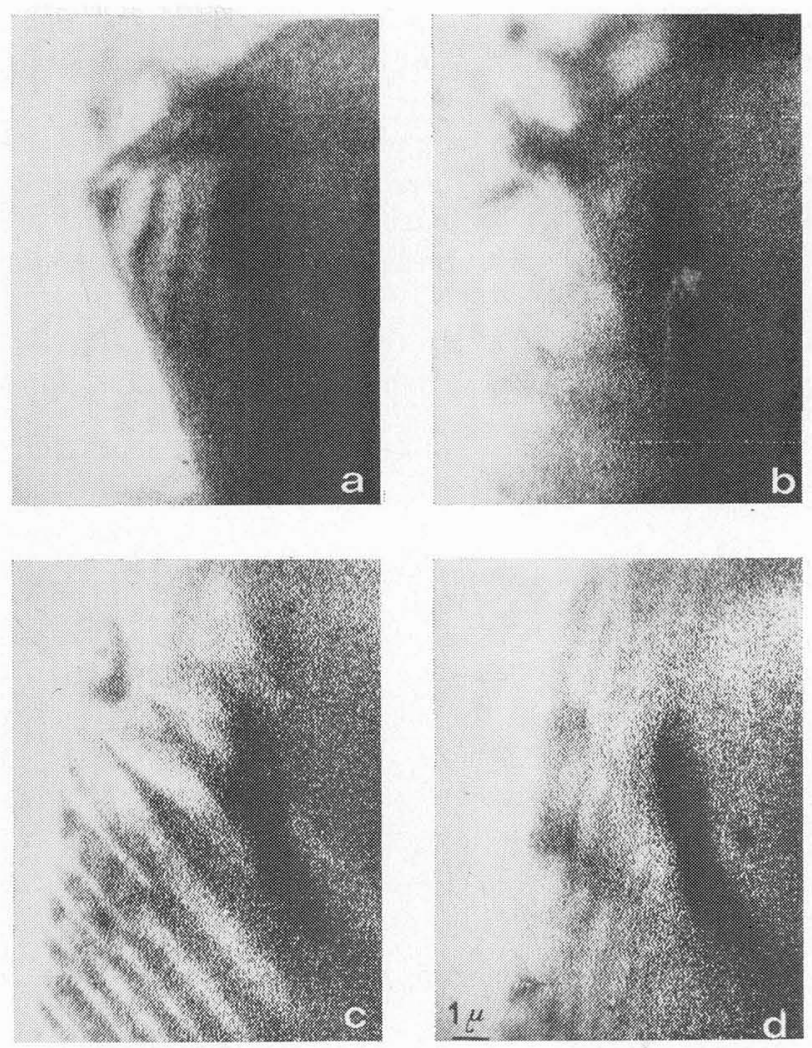

FIG. 4. - Transition AFI-M dans un cristal de $\mathrm{V}_{2} \mathrm{O}_{3}$, obtenue par augmentation de la température sous une tension de $1500 \mathrm{kV}$.

Après plusieurs transitions successives, les contraintes imposées provoquent des cassures dans le cristal. On a pu noter que celles-ci ne s'effectuent pas suivant les plans de séparation des domaines comme on pourrait s'y attendre. On peut également penser que les électrons de haute énergie perturbent nos expériences, ne serait-ce que par l'apport important d'énergie et la formation de défauts d'irradiation : la tension seuil étant largement dépassée. Nons avons limité ces perturbations en utilisant un faisceau d'électrons incidents de très faible intensité : de l'ordre de $1,6 \times 10^{-12} \mathrm{~A} / \mu \mathrm{m}^{2}$.

Le passage à la transition M-I (métal-isolant paramagnétique) a pu également être observé dans le cas d'un cristal dopé au chrome $\left(\mathrm{V}_{1-x} \mathrm{Cr}_{x}\right)_{2} \mathrm{O}_{3}$ avec $x=0,0055$. Il se traduit essentiellement par un changement brutal du contraste de l'image consécutif à une modification d'orientation du cristal, mais les interfaces entre phase métallique et phase isolante remarquées par Van Landuyt, Amelinckx et Remeika n'ont pas été observées. Dans le cas de $\mathrm{V}_{2} \mathrm{O}_{3}$ pur, la transition du second ordre M-I qui se produit entre 380 et $550 \mathrm{~K}$, n'a pas été mise en évidence au microscope électronique.

4. Conclusion. - L'ensemble de ces résultats appelle plusieurs remarques.

Dans le cas du sesquioxyde de vanadium pur ou 
dopé au chrome, la phase AFI est formée de domaines dont les configurations sont liées à des modèles cristallographiques simples.

Le changement de phase observé sous haute tension accélératrice fait apparaître un certain nombre d'états intermédiaires au voisinage de la température de transition. Cette évolution relativement lente obtenue sous $1500 \mathrm{kV}$ n'apparaît pas lorsqu'on opère sous tension usuelle $(100 \mathrm{kV})$ où la transformation est généralement brutale. A haute tension, les effets sont ralentis par diverses causes, en particulier par la présence certaine dans le cristal de défauts créés par irradiation aux électrons.

Il est probable que le changement brutal des caractéristiques physiques se produit lorsque les microdomaines apparaissent ou disparaissent subitement, c'est-à-dire essentiellement lorsque la symétrie de la maille passe de rhomboédrique à monoclinique ; la fluctuation des domaines n'entraînant que des modifications des propriétés physiques plus mineures.

\section{Bibliographie}

[1] Launay, J. C., Pouchard, M., Ayroles, R. et Jouffrey, B., Mater. Res. Bull. $10(1975) 559$.

[2] Launay, J. C., Pouchard, M. et Ayroles, R., J. Crystal Growth (sous presse).
[3] Van Landuyt, J., Amelinckx, S. and Remeika, J. P., Mater. Res. Bull. 7 (1972) 845.

[4] Hayashi, Y., van Landuyt, J. and Amelinckx, S., Phys. Stat. Sol. 39 (1970) 189. 